\title{
A Perfect Storm
}

\section{Brian Hainline, MD; Richard G. Ellenbogen, MD}

A perfect storm of events in the past decade has substantially increased public awareness of the evolving controversies surrounding concussion. A staggering number of service members have suffered traumatic brain injuries at home or in wars in Afghanistan and Iraq; most of these injuries are comparable with sport-related concussion. In addition, media coverage of concussions in prominent professional athletes and improved recognition of youth sports concussions have all led to the spotlight being focused on this previously neglected brain injury. Fortunately, this attention has produced an explosion in muchneeded concussion research, collaboration on sideline protocols, rule changes aimed at improving athlete safety, and enhanced coaching techniques that emphasize athlete health and safety.

Despite the important scientific research, passionate youth athlete advocacy, and activity-specific progress in on-field safety, a vocal segment of the media has aimed a concomitant increase in negative publicity at organized sports. The balance at times has tipped toward the perceived risk of contact-sport participation and away from the proven physical and cognitive benefits of exercise and team-sport participation. An unbalanced presentation of concussion risks in sports versus the benefits of such activities has caused some parents to question the overall value of athletic participation, despite overwhelming evidence that sport participation and exercise are cornerstones of health and wellness, both in individuals and within society.

Thus, we are at a crossroads in our public health mission as medical providers. The United States has become one of the most physically illiterate countries in the developed world. Physical literacy is having the ability, confidence, and desire to be physically active for life. Physical illiteracy correlates with poor overall health: specifically reduced neurologic health and cognitive development, increased risk of obesity and substance abuse, less academic success and earning potential, and higher health care costs. Physical illiteracy also prevents children and adults from actively receiving the many health benefits of sports, both individually and as team members.

As scientists, patient advocates, and public health activists, we have begun to educate our patients about the risks of concussion, but we must balance these discussions with the broad and far-reaching benefits of exercise and sports, both for individuals and communities. At any level of sport (and in all forms of human activity for that matter), there will always be risk. In a free society, judgment about pursuing these personal choices should be based on unbiased scientific information and common sense. To date, we have done a poor job in providing the scientific equipoise necessary for our patients to make truly informed decisions about sports. The questions surrounding the risks of sport have transcended the scientific realm and become an existential threat to the survival of these activities. A rapidly evolving state of scientific evidence should be presented by scientists in a thorough and balanced manner, a feat now rendered nearly impossible by the highly volatile and emotional debate in the media.

What are the concussion-specific concerns for athletes? Despite considerable scientific progress, we remain in the infancy of our knowledge about some of the most profound concussion-related questions. In part, this stems from a lack of consensus as to the precise definition of concussion. Of approximately 43 working definitions of concussion, only 1 is evidence based, and none provide any specific information regarding brain localization, biomechanics, or pathophysiology. In other words, concussion definitions describe a generalized state of disturbed brain pathophysiology resulting from an impulsive force to the head. However, this disturbed neurologic state has been defined for all concussed individuals in a manner that suggests concussion is a homogeneous clinical entity. It is not. Concussion describes a broad spectrum of conditions. Emerging evidence tells us that concussions are characterized by diverse symptoms and functional impairments that may result in different recovery trajectories. Furthermore, such diverse symptoms and impairments may require treatment strategies beyond the gold standard of rest and gradual return to activity as tolerated.

Beyond concussion lies the even more poorly defined risk of repetitive head-impact exposure, which is separate from the impulsive force to the head that presents as a clinical manifestation of concussion. Stated differently, does repetitive head-impact exposure in women's ice hockey (eg, from falls to the ice or head impacts with the boards) lead to functional or structural brain changes that are distinct from those of single or multiple concussions? Although our current challenge is to precisely and reproducibly define forces to the head through accelerometer technology, we cannot, as yet, provide an accurate risk assessment for repetitive head-impact exposures.

Because our definition of concussion encompasses a spectrum of conditions and because the hypothesis that repetitive head-impact exposure represents such an enormous risk to all athletes, clinicians are presently unable to answer some of the most compelling concussion questions facing us: 
- What are the incidence and prevalence of long-term neurologic, psychological, and structural sequelae of concussion?

- What are the long-term neurologic, psychological, and structural sequelae associated with single versus repetitive head impacts?

- Are some individuals more susceptible to long-term risks than others, and if so, can we identify them?

- What are the pathophysiologic mechanisms underlying the long-term sequelae of concussion and repetitive headimpact exposure?

- What are the natural histories of the chronic sequelae of concussion and repetitive impacts? Specifically, is there a continuous and progressive course or a delayed onset with a defined latency period?

- What are the engineering advances in helmets, turf, and safety equipment that can lower the rate and risks of concussion in contact-sport athletes?

We must try to answer such questions within an evidence-based neurologic and psychological model of multiple domain predictor variables. Emerging evidence tells us that there are neurobiological and psychosocial predictors of concussion recovery:

- Preinjury factors, including genetics and neurologic vulnerabilities;

- Trauma burden, including injury severity - from both singular and repetitive impacts - and polytrauma;

- Biomarkers of injury, including structural and functional brain injury and blood and cerebrospinal byproducts of brain injury;

- Psychological function, including premorbid and postinjury comorbidities such as depression;

- Environmental factors, including social support, life stressors, and iatrogenesis;

- Motivational factors, including expectation of recovery and secondary gain.

Several large-scale efforts are underway to address these knowledge gaps. The US Veterans Administration and the Department of Defense have jointly funded the Chronic Effects of Neurotrauma Consortium, which uses a cross-sectional design that focuses on cohorts of military personnel with prior exposure to brain injury. The General Electric-National Football League Head Health Initiative is funding multiple studies that advance concussion diagnosis and management. The National Collegiate Athletic Association and the Department of Defense are conducting a multisite, prospective, longitudinal study of the natural history of acute concussion and neurobiological recovery that is already the largest study ever conducted in concussion research. This study will not only address concussion recovery but will also assess whether there is a continuum of clinical and neurobiological presentations across the spectrum of athletes in noncontact and contact sports who have no head-impact exposure, repetitive head-impact exposures, or clinical concussion. To further advance concussion science, the brain magnetic resonance imaging protocol is synchronized with Transforming Research and Clinical
Knowledge in Traumatic Brain Injury (TRACK-TBI), a multicenter study that collects and analyzes detailed clinical and magnetic resonance imaging data from patients with traumatic brain injuries across the country. Finally, recognizing that despite the great deal of media attention to chronic traumatic encephalopathy and its possible relationship to contact sports, even though the scientific study of this disease is still young, the National Institutes of Health has funded the Diagnostics, Imaging, And Genetics Network for the Objective Study and Evaluation of Chronic Traumatic Encephalopathy (DIAGNOSE CTE) Research Project. This 7-year, multicenter, multidisciplinary longitudinal study is designed to develop and refine methods of diagnosing chronic traumatic encephalopathy during life and to examine risk factors for the condition.

Although it is comforting to know that the science of concussion is making important advances, we must be ever mindful of the frontline management of patients with concussion. We believe that the athletic trainer (AT) offers a realistic and professionally sound solution to both on-field and first-line off-field management of players with sport-related concussion. Athletic trainers have played a pivotal role in advancing concussion science and clinical care, and they are uniquely trained to recognize and manage patients with concussions and head injuries in addition to other sport-related injuries. Indeed, the National Collegiate Athletic Association has defined the primary athletics health care providers as the AT and team physician and has stated that these providers have autonomous authority to make medical and return-to-play decisions for all student-athletes. In other words, the AT is at the foundation of medical care delivery in collegiate sports, including concussion diagnosis and management. Part of the multidisciplinary team of medical experts at the professional level, ATs play an important role in injury management, including concussion. Despite the clearly defined roles of health care providers in collegiate and professional sports, select state legislators are sending murky and dangerous signals about ATs. In California, ATs have no state accreditation or certification oversight, and there has been a recent push to decertify ATs in several other states. We believe this is a short-sighted, potentially deleterious path for athletes and active citizens throughout our nation.

At the youth level, ATs are too frequently absent from high-risk contact-sport practices and competitions. A lack of funding and, thus, infrastructure support for youth athletes often leaves them without this cost-effective and experienced health and safety resource. We believe that ATs are the foundation to ensure proper first-line concussion diagnosis and management for youth, high school, collegiate, and professional athletes. We hope that, just as scientific advances in concussion research will evolve, a national infrastructure that validates the role of ATs in providing health care to patients with sport-related injuries will also evolve.

As our understanding of concussive injuries improves, concussion management must be based on emerging 
evidence that has been established by the scientific community. This special edition on concussion in the Journal of Athletic Training is a positive step toward consolidating such developing evidence and presenting it in a manner that will greatly benefit the sports medicine community and society at large.

Editor's note: Brian Hainline, MD, is the Chief Medical Officer of the National Collegiate Athletic Association and clinical professor of neurology at the Indiana University School of Medicine and the New York University School of Medicine. Richard G. Ellenbogen, $M D$, is the Co-Chair of the National Football League Head, Neck, and Spine Committee and professor and Chairman of the Department of Neurological Surgery at the University of Washington School of Medicine. 